Research

\title{
Feasibility of an adjunctive cognitive task in the treatment of posttraumatic stress disorder
}

\author{
Amalia Badawi $^{1}$ (D) Zachary Steel ${ }^{2,3,4}$ (D) $\cdot$ Christopher Mahoney ${ }^{5} \cdot$ David Berle $^{1,2,6}$ (D) \\ Received: 22 October 2021 / Accepted: 14 December 2021 \\ Published online: 20 December 2021 \\ (c) The Author(s) 2021 OPEN
}

\begin{abstract}
Visuospatial cognitive tasks that influence memory reconsolidation may be of benefit in reducing intrusive memories for traumatic events when used as an adjunct to trauma-focused psychotherapy. We conducted a feasibility assessment of a protocol that involved the use of a visuospatial cognitive task, the Tetris intervention, alongside routine exposurebased treatment for posttraumatic stress disorder (PTSD). Participants were inpatients attending for PTSD treatment at a psychiatric hospital. The Tetris intervention was administered on three occasions when imaginal exposure had formed part of the treatment session. Using a phone app, participants also monitored intrusive memories over a 3-week period. Feasibility outcomes were fully met for the demand, implementation, practicality and adaptability criteria. Only a single criterion was not met for each of the acceptability and implementation criteria. Limited-efficacy testing outcomes are also discussed. Overall, the findings from our feasibility study indicated viability of the protocol, which involved implementation of the Tetris intervention alongside routine exposure-based treatment for PTSD, in a clinical inpatient setting.
\end{abstract}

Keywords Feasibility $\cdot$ Inpatient $\cdot$ Intrusive memories $\cdot$ Posttraumatic stress disorder $\cdot$ Tetris

\section{Introduction}

The scientist-practitioner model of psychological practice demands that research outcomes be assessed for feasibility of implementation in clinical settings in order to support translation into routine practice [1]. A growing area of investigation has been the use of visuospatial cognitive task interventions involving computer gameplay to disrupt the trajectory of intrusive memories following exposure to trauma. The most commonly used task has been the three-part Tetris intervention comprising mental rotation practice, a memory reactivation cue, and Tetris gameplay. Despite promising outcomes with non-clinical $[2-6]$, potentially clinical $[7,8]$, and clinical $[9,10]$ samples, the use of the Tetris intervention alongside routine treatment for posttraumatic stress disorder (PTSD) presents a translational gap that has not been investigated.

Intrusive memories, the repeated and involuntary memories arising after exposure to a trauma event, are a key feature of PTSD. Although trauma-focused therapy is the globally recommended treatment for PTSD $[11,12]$ estimates indicate that one-third of people with PTSD will not benefit from such treatment, with a further third likely to only make moderate gains [13]. Given the predictive role of intrusive symptoms in the development of PTSD [14, 15] adjunct interventions that target intrusive memories hold promise for optimising existing treatments following trauma exposure.

David Berle, David.Berle@uts.edu.au | ${ }^{1}$ Graduate School of Health, University of Technology, Sydney, Australia. ${ }^{2}$ School of Psychiatry, University of New South Wales, Sydney, Australia. ${ }^{3}$ St. John of God Health Care, Sydney, Australia. ${ }^{4}$ Black Dog Institute, Randwick, Australia. ${ }^{5}$ South Coast Private Hospital, Wollongong, Australia. ${ }^{6}$ Discipline of Clinical Psychology, Graduate School of Health, University of Technology Sydney, Broadway, PO Box 123, Sydney, NSW 2007, Australia.

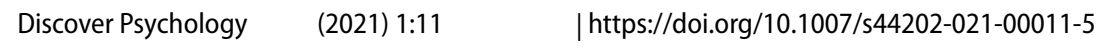


Initial studies with non-clinical samples used the Trauma Film Paradigm (TFP) [4] to generate intrusive memories, with administration of the Tetris intervention then taking place in the memory consolidation phase. Memory consolidation refers to the transition of memories from short- to long-term storage [16] and is thought to take place within 6-h of exposure to an event, though memories can later be rendered labile through reactivation $[17,18]$ Subsequent re-stabilisation of the memory following re-activation is referred to as memory reconsolidation $[19,20]$. In the context of psychological therapies, the recall of a traumatic event during an exposure-based therapy could be considered the reactivation cue that precipitates reconsolidation of a memory in the hours following the exposure therapy session.

Non-clinical studies have used the Tetris intervention during the memory consolidation phase at differing times points after the TFP was viewed; that is at 10- and 30-min [4] at 30-min and 4-h [5], and at 4-h [2] post-viewing. In these studies, the Tetris intervention was associated with decreases in intrusive memories for the film when compared to control conditions. These findings from trauma analogue samples were then extended in two key ways: (1) application of the intervention in the memory consolidation phase following direct exposure to potentially traumatic events and (2) application of the intervention during memory reconsolidation.

Two studies involved administration of the Tetris intervention in the memory consolidation phase following exposure to a potentially traumatic event. lyadurai et al. [8] administered the task in a hospital emergency setting to people who had witnessed or been involved in a motor vehicle accident. Horsch et al's [7] study involved women who had given birth via an emergency caesarean procedure. In both of these studies, participants in the intervention condition reported fewer intrusive memories at one-week follow-up when compared to participants in the control conditions. Importantly, these studies not only demonstrated task efficacy, but also the feasibility of administering the task with distressed individuals in time-pressured environments.

Four studies have reported on outcomes following application of the Tetris intervention as part of memory reconsolidation processes. Findings of Tetris intervention efficacy for reducing intrusive memories following exposure to a TFP have been demonstrated with participants at 24-h [6] and 4-days [3] post-viewing. Decreases in intrusive memory frequency following use of a Tetris-based intervention have also been reported for people presenting for PTSD [10] and in a case-series involving four people from refugee backgrounds [9] when brief reminder cues were used prior to gameplay. Despite preliminary demonstrations of efficacy, in that the frequency of intrusive memories decreased following use of a Tetris intervention for trauma-exposed individuals $[9,10]$, it remains unclear how such an intervention might best be deployed for use by clinicians in the context of a routine treatment program for PTSD.

As no previous study has integrated the Tetris intervention alongside routine exposure-based therapy, we aimed to explore the feasibility of implementing a Tetris-based intervention as an adjunct to standard treatment for PTSD in an inpatient setting. Trauma-focused therapy approaches involve memory reactivation via imaginal exposure [11, 12]. Therefore, our Tetris intervention involved mental rotation practice followed by imaginal exposure as the reactivation cue prior to Tetris gameplay. Our broader feasibility investigation involved an observational approach designed to test whether the protocol used in a non-clinical sample [2] could be applied to a real-world psychiatric hospital setting with minimal interference to routine treatment.

\section{Method}

\subsection{Participants}

Participants were 23 adults attending an individual inpatient PTSD treatment program. Participants were predominantly from military, first responder, and occupational injury backgrounds and were referred to the program by their treating psychiatrist. PTSD was assessed as part of the intake process at the hospital using the Clinician-Administered PTSD Scale for DSM-5 (CAPS-5) [21]. In order to access treatment, and therefore to participate in this study, participants had to be English speakers over the age of 18 and to have experienced the index trauma in adulthood. Exclusion criteria for participation in this study were consistent with those of the hospital for admission and included nil current or previous chronic psychosis, problematic substance use issues or need for detox, active self-harm or intention to suicide.

Ethical approval was obtained from the Medical Advisory Committee at the hospital site, the Human Research Ethics Committee at the University of Technology Sydney (Reference Number: ETH17-1912), and the Departments of Defence and Veterans' Affairs Human Research Ethics Committee (Reference Number: 031-18). 


\subsection{Tasks and materials}

\subsubsection{The Tetris intervention}

2.2.1.1 Memory reactivation Memory reactivation was considered to have taken place when a participant's traumafocused session consisted of at least 15-min of imaginal exposure.

2.2.1.2 Tetris Blue Planet Software specifically adapted Tetris for use in this body of research (UTS Research Version) [22]. The computer game involves seven blocks of differing shapes and colours falling from the top of the screen, with the aim being that the player rotate the shapes to create horizontal lines in order to earn points and clear the lines from the screen. Participants were able to see the three upcoming blocks and were instructed to also keep their attention on these blocks and imagine how they would rotate them to make the lines.

Standard Tetris mode speeds up the rate of falling blocks and terminates the game when the blocks fill the screen. In the modified version, the rate of blocks dropping did not exceed level 5 and the screen automatically emptied to halfway once it filled up to allow a more continuous gameplay experience. Tetris was played on a Lenovo tablet with 10-inch screen size, which allowed participants to rotate the blocks by tapping the screen and to drag the blocks sideways or downward by pressing on the block and moving it in the desired direction. The automated Tetris gameplay task was extended from 10-min, per the protocol by Badawi et al. [2], to 12-min in order to be consistent with James et al.'s [6] study, which was conducted in the memory reconsolidation phase.

\subsubsection{Diary for intrusive memory recording}

Participants downloaded the ExpiWell app [23] on to their Android or IOS phone to track intrusive memories across days $0-21$. On day 0 , participants were asked to complete the survey at 5:00 pm only, then three times each day on days 1-21 at 8:30 am, 12:30 pm, and 5:00 pm, for a total of 64 time points. At each time, participants received an automated reminder that the survey was available. A follow-up notification was sent if the survey had not been completed within 30-min. The survey asked about intrusive memory frequency, strength of relationship to event attended to in the most recent therapy session on a sliding scale from 0 to 100 , emotional valence on sliding scales from 0 to 10, manner in which memories were triggered, vantage perspective, and a comment section asked about the memories. One participant who chose not to download the phone app was issued with a paper-and-pen diary and asked to also record intrusive memory information three times each day.

\subsubsection{Visual analogue scales}

Before and after engaging in each Tetris gameplay session, participants were provided with a sheet asking them to rate levels of how sad, hopeless, fearful, horrified, and depressed they were feeling on a scale from 0 (not at all) to 10 (extremely) by making a mark along the continuum provided. The post-task scales also asked about ability to concentrate on the task and enjoyment level on a continuum from 0 (not at all) to 10 (very much).

\subsubsection{Clinician rating scales}

On each of the three occasions that a participant played Tetris, the clinician was asked to report on the session number as part of the overall number of therapy sessions, length of imaginal exposure component for that session, the percentage of time spent in the session on different approaches (e.g., psychoeducation, imaginal exposure), with the total needing to add up to $100 \%$, and to record the participant's Tetris score. 


\subsubsection{Self-report measures}

2.2.5.1 Demographics Participants completed a questionnaire to obtain age, gender, occupation, first language, medication use, and physical ailment information on day 0 only.

2.2.5.2 Posttraumatic Stress Disorder Symptoms The Posttraumatic Stress Disorder Checklist for DSM-5 (PCL-5) $[21,24]$ is a 20-item scale used to assess PTSD severity overall and at the cluster level using a 5 -point Likert scale $(0=$ not at all to $4=$ extremely) on days 0 and 21. Strong reliability has previously been reported for this scale (Cronbach's $a=0.93)$ [25].

2.2.5.3 Re-experiencing The Impact of Events Scale-Revised (IES-R) [25] 8-item Intrusions subscale was used to assess subjective distress of traumatic events using a 5 -point Likert scale $(0=$ not at all to $4=$ extremely) on days 0 and 21. High levels of internal consistency have been reported for this scale $(a=0.86)$ [26].

2.2.5.4 Depression and anxiety The Hospital Anxiety and Depression Scale (HADS) [27], which was developed in a hospital setting, uses a 4-point Likert scale (0-3) to assess symptom levels on days 0 and 21. A review of 747 studies reported high subscale reliability means of Cronbach's $a=0.83$ for anxiety and Cronbach's $a=0.82$ for depression [28].

2.2.5.5 Psychological distress The Kessler Psychological Distress Scale (K10) [29] was used to assess general levels of psychological distress using a 5-point Likert scale ( $1=$ none of the time to $5=$ all of the time) on days 0 and 21 . In a large sample $(n=6700)$, high levels of internal consistency have been reported (Cronbach's $a=0.88)$ [30].

2.2.5.6 Anger The Dimensions of Anger Reactions-5 (DAR5) [31] was used to assess levels of anger at days 0 and 21 using a 5-point Likert scale ( $1=$ none of the time to $5=$ all of the time). Forbes et al. [32] have reported strong reliability (Cronbach's $a=0.89$ ) in a trauma-exposed sample.

\subsection{Treatment}

Treatment-as-usual for patients presenting with PTSD involved trauma-focused cognitive behavior therapy for three to four sessions a week, with most sessions including a component of imaginal exposure. Patients generally attended for 3-5-weeks at the hospital, with the duration being dependent on factors such as rate of recovery and the strength of their support system in place following discharge. Given that individual treatment for PTSD demands variability in frequency and duration of treatment, participants in the current study took part in the Tetris intervention on the first three occasions of therapy that involved imaginal exposure to support consistency of implementation across participants.

\subsection{Procedure}

On day 0 , usually the day following admission to the hospital, participants met with the researcher who explained the study. Participants then provided informed consent and demographic information, and practiced the Tetris task involving mental rotation practice and gameplay for two minutes. Participants were provided with verbal instructions on the task and asked to repeat them to facilitate understanding of the task requirement. Participants also completed hospital and study-specific measures. An explanation of intrusive memories was then given, which included questions to assist with checking participant understanding. Participants downloaded the ExpiWell app on to their personal phone and instructions were provided for its use. Where necessary, a paper-and-pen diary was issued at this time instead. The researcher then prepared a folder for the treating clinician to assist with management of documents and provided the clinician with a tablet for use during the 21-day period.

Participants engaged in treatment-as-usual for the next 21-days. Within this period, participants completed the Tetris intervention on three occasions. Each occasion required that the therapy session had involved imaginal exposure for 15-min or more, and completion of visual analogue scales before and after Tetris gameplay. The treating clinician also completed a rating sheet each time. Participants used a phone app to monitor intrusive memories on the evening of day 0 and three times daily across days $1-21$. Post-intervention measures were administered on day 21 . Figure 1 outlines the participant flow and study protocol. 


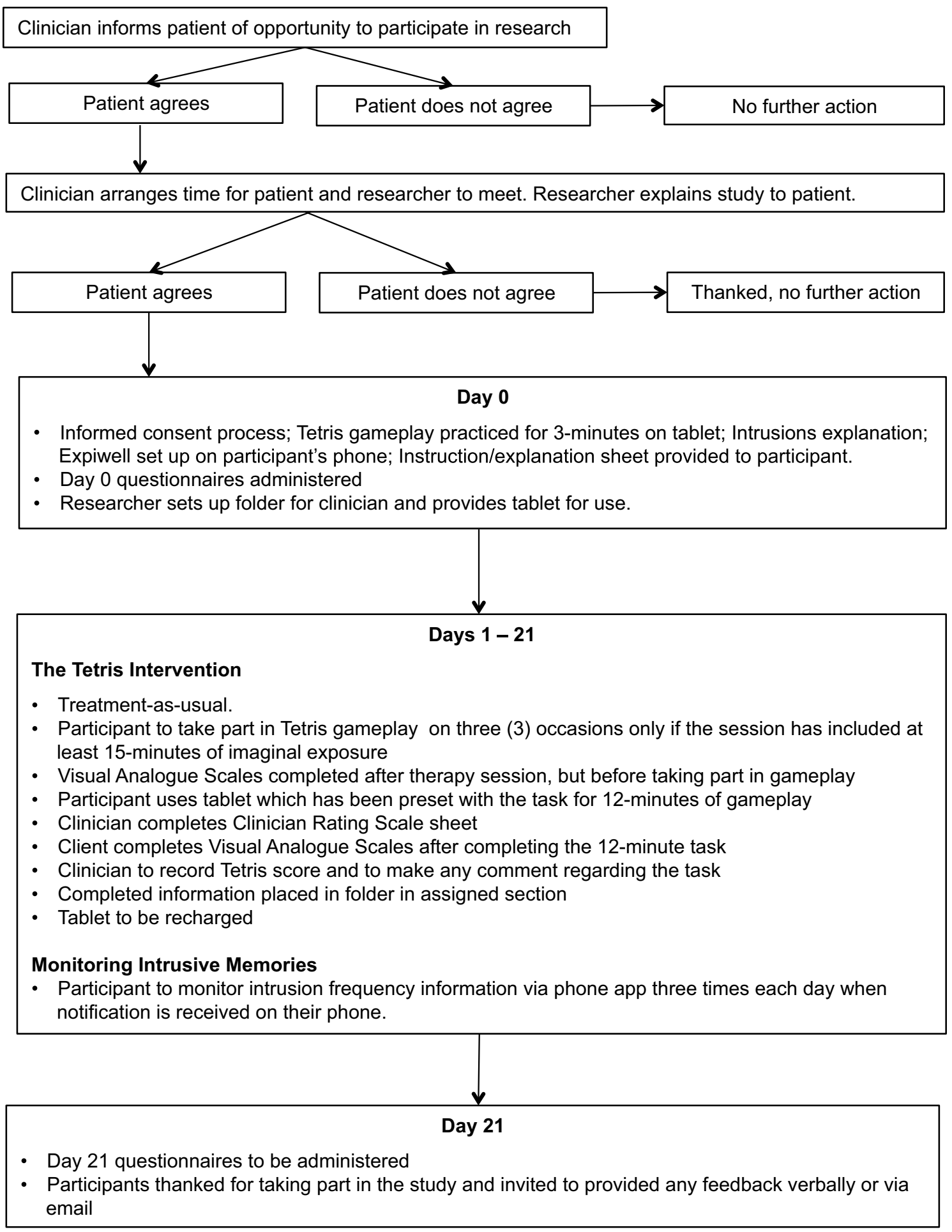

Fig. 1 Participant flow and study protocol

\subsection{Analysis}

Feasibility criteria consistent with those defined by Bowen et al. [33] were used to assist with identifying and refining an intervention protocol for use in a clinical PTSD population. Table 1 outlines these criteria (columns 1-3) and 


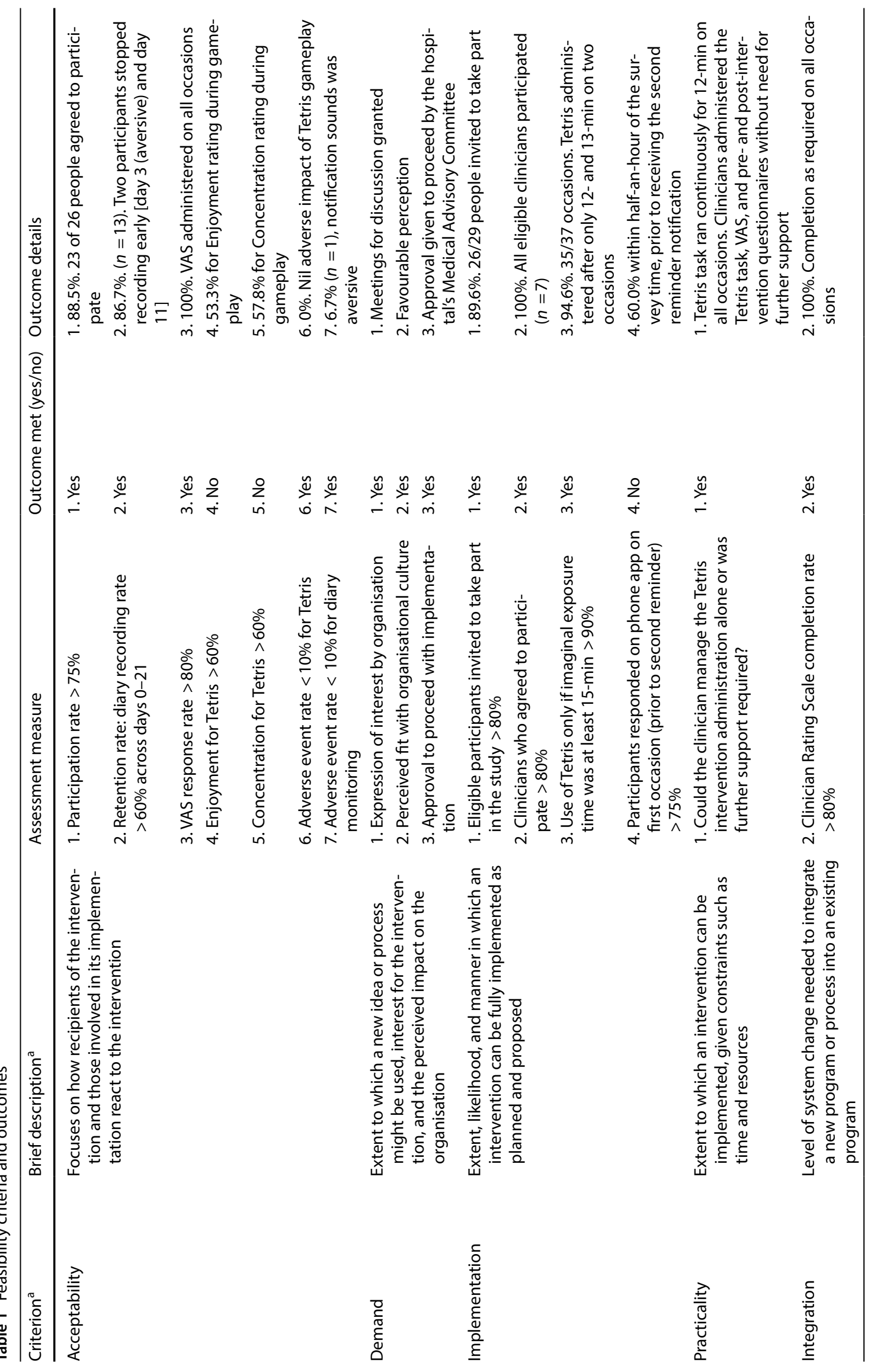




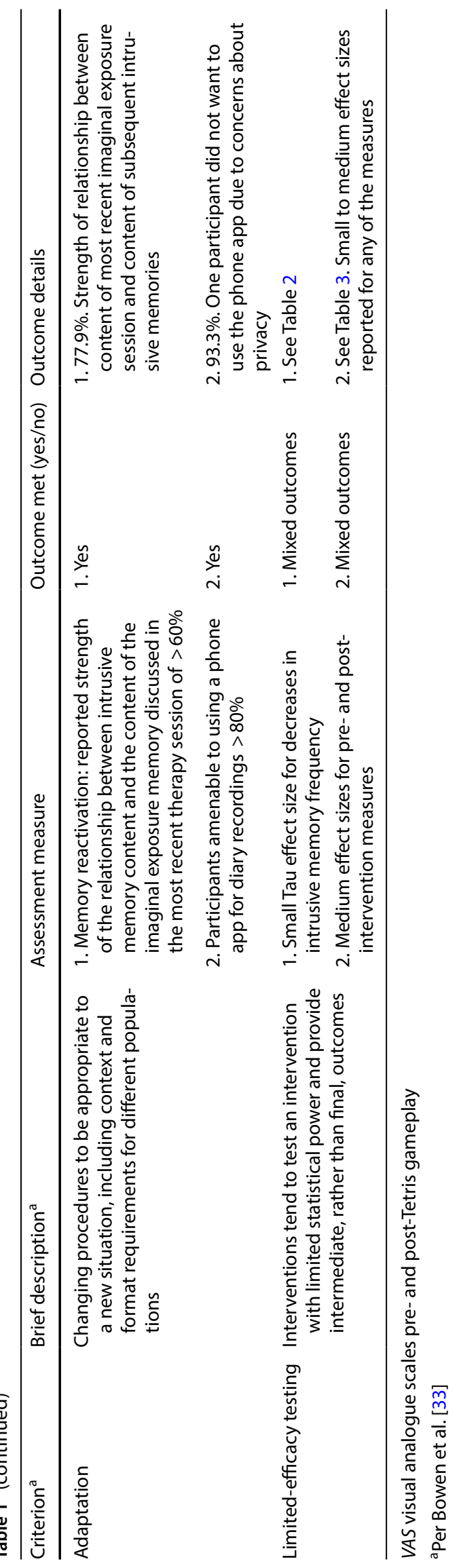


describes the way in which each criterion was assessed in the present study. Feasibility outcomes are reported in a descriptive manner, except for those pertaining to efficacy testing.

The IBM Statistical Package for Social Sciences (SPSS) Version 27 was used to calculate percentages and effect sizes using Hedge's $g$ for the self-report measures of trauma, re-experiencing, depression, anxiety, psychological, distress, and anger. Hedge's $g$ was used due to the small sample size $(<20)$, and effect size values of $0.2,0.5$ and 0.8 were considered small, medium, and large respectively [34]. Thresholds for task enjoyment and concentration were set at $60 \%$ based on our previous study [2], where the mean enjoyment percentage for Tetris gameplay was $60.93(S D=28.10)$.

A single-case experimental design was employed to allow for limited-efficacy testing of changes in intrusive memory frequency [35]. Tarlow's [36, 37] baseline corrected Tau calculator was used in order to obtain effect sizes and significance values for intrusive memory frequency over the course of the intervention. An ABB design was used to assess outcomes from Time 1 to Time $2\left(A B_{1}\right)$, and Time 1 to Time $3\left(A B_{2}\right)$. Time 1 was the period prior to any Tetris gameplay; Time 2 was the period after the first gameplay but before the second gameplay; and Time 3 was the period after the third gameplay.

\section{Results}

Of the 23 participants who consented to participate in the study, eight did not continue on in the study due to changes in treatment approach that meant imaginal exposure would not be used $(n=5)$ or early discharge occurred prior to task engagement $(n=3)$. As such, outcomes are reported for 15 participants.

\subsection{Feasibility outcomes}

The feasibility outcomes are summarized in Table 1 (columns 4 and 5). For Acceptability, the key measures of participation (88.5\%) and retention (86.7\%) rates exceeded the thresholds set. Only the outcome levels of enjoyment and concentration for Tetris gameplay did not meet the set thresholds, with levels reported at $53.3 \%$ and $57.8 \%$ respectively. Importantly, the adverse event rate for Tetris gameplay was $0 \%$.

All outcomes were met for Demand, Practicality, and Adaptability. Across these three areas, particular focus is drawn to outcomes showing that the clinicians were able to implement the intervention alone without the need for additional support, the strength of relationship between memory content in the therapy session and the intrusive memories that were reported on using the phone app (77.9\%), and the high rate of uptake for monitoring using a phone app (93.3\%).

Under the criterion of Implementation, high levels of participation by patients (89.6\%) and clinicians (100\%), and adherence to the protocol relating to imaginal exposure length (94.6\%) were reported. The only measure that was not met was in relation to Implementation was the time of diary completion with participants responding within 30-min of receiving notification to complete the survey $60 \%$ of the time.

Efficacy outcomes indicated small to medium effect sizes for the repeated measures for trauma, re-experiencing, depression, anxiety, psychological distress, or anger over the course of the 21-day period. Data are reported in Table 2. The single-case experimental outcomes for intrusive memory frequency are shown in Table 3 for the nine participants who provided sufficient data for establishment of a baseline period prior to application of the intervention. Data for the participant who used the paper-and-pen diary were not useable due to the use of descriptive terms rather than count data. Small effect sizes were reported for decreases in intrusive memory frequency at both time points $\left(A B_{1}: n=2 ; A B_{2}\right.$ : $n=5)$.

\section{Discussion}

Dissemination of research findings through clinical implementation is essential for bridging translational gaps between research and clinical utility, in order to continuously improve mental health outcomes [38]. Our current study aimed to assess the feasibility of utilizing a previously deployed protocol [2] that included a visuospatial cognitive task-the Tetris intervention-in a hospital inpatient setting for people presenting with PTSD. Although the Tetris intervention has previously been investigated in relation to intrusive memory trajectory following exposure to trauma $[9,10]$, to our knowledge, this is the first study to utilize the Tetris intervention as an adjunct treatment alongside routine treatment for PTSD.

The protocol of the present study was based on that of our previous study [2] and was extended to investigate whether use of imaginal exposure as the reactivation cue, an automated Tetris gameplay task, and monitoring of intrusive 
Table 2 Effect size by participant for changes in intrusive memory frequency

\begin{tabular}{lllll}
\hline Participant & Time & Tau effect size & $p$ value & $S E_{\text {Tau }}$ \\
\hline 1 & $\mathrm{AB}_{1}$ & 0.13 & 0.747 & 0.44 \\
1 & $\mathrm{AB}_{2}$ & -0.25 & $0.048^{*}$ & 0.20 \\
2 & $\mathrm{AB}_{1}$ & -0.10 & 0.561 & 0.26 \\
2 & $\mathrm{AB} \mathrm{B}_{2}$ & $\mathrm{~N} / \mathrm{A}$ & $\mathrm{N} / \mathrm{A}$ & $\mathrm{N} / \mathrm{A}$ \\
3 & $\mathrm{AB} \mathrm{B}_{1}$ & 0.77 & 0.110 & 0.37 \\
3 & $\mathrm{AB}_{2}$ & -0.02 & 0.955 & 0.30 \\
8 & $\mathrm{AB}_{1}$ & 0.47 & 0.080 & 0.35 \\
8 & $\mathrm{AB}_{2}$ & -0.60 & $0.011^{*}$ & 0.28 \\
9 & $\mathrm{AB}{ }_{1}$ & $\mathrm{~N} / \mathrm{A}$ & $\mathrm{N} / \mathrm{A}$ & $\mathrm{N} / \mathrm{A}$ \\
9 & $\mathrm{AB}_{2}$ & -2.65 & $0.032^{*}$ & 0.20 \\
10 & $\mathrm{AB}_{1}$ & -0.41 & 0.112 & 0.33 \\
10 & $\mathrm{AB}_{2}$ & -0.20 & 0.19 & 0.23 \\
12 & $\mathrm{AB}_{1}$ & -0.44 & 0.109 & 0.35 \\
12 & $\mathrm{AB}_{2}$ & -0.44 & $0.003^{* *}$ & 0.20 \\
15 & $\mathrm{AB}_{1}$ & -0.52 & $0.001^{* * *}$ & 0.20 \\
15 & $\mathrm{AB}_{2}$ & -0.39 & $0.008^{* * *}$ & 0.22 \\
16 & $\mathrm{AB}_{1}$ & -0.46 & $0.000^{* * *}$ & 0.18 \\
16 & $\mathrm{AB}_{2}$ & $\mathrm{~N} / \mathrm{A}$ & $\mathrm{N} / \mathrm{A}$ & $\mathrm{N} / \mathrm{A}$ \\
\hline
\end{tabular}

+ Participant completed Tetris gameplay 3 days in row. As such, no analyses for Time $A B_{1}$ were conducted $N / A$ not applicable

${ }^{* * *} p<0.001 ;{ }^{* *} p<0.01 ;{ }^{*} p<0.05$

\begin{tabular}{|c|c|c|c|c|c|c|c|c|}
\hline & \multirow[t]{2}{*}{$n$} & \multicolumn{2}{|c|}{ Pre-treatment } & \multicolumn{2}{|c|}{ Post-treatment } & \multicolumn{2}{|c|}{ Difference score } & \multirow[t]{2}{*}{ Hedge's $g$} \\
\hline & & Mean & $S D$ & Mean & $S D$ & Mean & $S D$ & \\
\hline PCL-5_Total & 9 & 59.00 & 8.00 & 50.89 & 17.51 & -8.11 & 16.31 & 0.47 \\
\hline IES-R & 9 & 30.56 & 5.36 & 28.00 & 6.38 & -2.56 & 6.84 & 0.37 \\
\hline HADS_Depression & 8 & 12.25 & 4.86 & 10.67 & 4.03 & -1.88 & 4.70 & 0.38 \\
\hline HADS_Anxiety & 9 & 15.00 & 2.65 & 13.11 & 4.60 & -1.89 & 4.76 & 0.38 \\
\hline K10 & 10 & 36.30 & 9.18 & 30.80 & 9.88 & -5.50 & 8.76 & 0.60 \\
\hline DAR-5 & 9 & 16.56 & 5.13 & 14.11 & 5.11 & -2.44 & 3.61 & 0.65 \\
\hline
\end{tabular}

$n=13$

PCL-5 posttraumatic stress disorder checklist for DSM-5; IES- $R$ impact of events-revised (re-experiencing subscale only); HADS Hospital Anxiety and Depression Scale; K10 Kessler Psychological Distress Scale; DAR5 Dimensions of Anger Reactions Scale- 5

memories via a phone app would be viable in a clinical sample. Feasibility of the current study was based on the criteria outlined by Bowen et al. [33], with overall indications suggesting that the protocol was feasible for application in a sample of participants presenting for PTSD in a hospital environment when the Tetris intervention was used as an adjunct to treatment as usual.

The Tetris intervention involves mental rotation practice and memory reactivation prior to gameplay. An important difference between the current clinical study and previous non-clinical studies that have used a TFP to generate intrusive memories as part of memory reconsolidation $[3,6]$ was the use of imaginal exposure based on autobiographical memory as the reactivation cue. The relatively high percentage reported for the relationship between intrusive memory content and the therapy session content coupled with the small effect sizes for intrusive memory frequency from Time 1 to Time 3 for some participants, suggests that it may not be necessary to target specific memories prior to engagement in Tetris gameplay. This premise differs in methodology from that of Kessler et al. [10] where specific memories were reactivated through a writing process prior to gameplay. 
Should imaginal exposure that occurs as part of standard treatment for PTSD prove sufficient to serve as the reactivation task, practicality and integration of the Tetris intervention into treatment is likely to be enhanced. In the current study, clinicians provided participants with the tablets for Tetris gameplay at the end of a therapy session that had included at least 15-min of imaginal exposure. This accessibility, coupled with a Tetris version that did not require monitoring or re-setting, facilitated ease of implementation. Clinicians reported that they used the 12-min period of gameplay to complete their rating form and to attend to administrative tasks such as session notes. We note that if the Tetris intervention is used solely for clinical purposes, use of ratings such as the VAS and Clinician Rating Scales would not be required, further facilitating ease and practicality of implementation.

A key component of any feasibility study is investigation of acceptability of the protocol and tasks [33]. Acceptability in this study was indicated by high levels of retention that were measured by diary recordings across the 21 days and completion of the VAS on all occasions. One participant experienced the phone notifications as aversive due to idiosyncratic correlations between their device and a trauma event. As such, future studies that seek to electronically monitor memories or send reminders via phone for completion of paper diaries may benefit from ensuring that the chosen monitoring format is appropriate for clinical participants.

Strengths for diary monitoring via electronic means, such as a phone app, are indicated given that data for the participant who used the paper diary were not useable for statistical analysis due to descriptive responses, and that our set threshold was not met for participants completing the diary within 30-min of the assigned time. Using an app would likely be beneficial in ensuring retrospective diary entries are not made outside of circumscribed windows for recording and for ensuring data are useable.

Unexpectedly, levels of task enjoyment for Tetris gameplay were lower than anticipated given outcomes of previous studies that showed that gameplay was perceived as relatively enjoyable [22], pleasant [3], and easy [8]. It is possible that Tetris gameplay was perceived as less enjoyable given that the participant would have just completed an entire traumafocused therapy session that included recall of a trauma event. This is further indicated by the lower than expected concentration levels, which may be indicative of mental and/or emotional fatigue following the session.

Formal hypothesis testing for efficacy of an intervention is generally not recommended in feasibility studies given the tendency toward small sample sizes [39]. As such, consistent with limited-efficacy testing recommendations [33], we calculated effect sizes only. Two participants reported a small effect size for intrusive memory decline from Time 1 to Time 2, in comparison to five participants for Time 1 to Time 3 . These findings suggest promise in regard to using the Tetris intervention alongside treatment-as-usual for PTSD, however the optimal frequency of intrusive memory recording in clinical populations requires further investigation in order to ascertain the ideal balance between capturing data accurately and levels of participant burden.

Feasibility of the protocol used for the present study appeared to be supported for use of the Tetris intervention alongside routine treatment for PTSD, as indicated by the successful outcomes for the majority of assessable items. Despite the threshold for diary response on the first occasion not being met, the overall retention rate was very high in the study, indicating feasibility of electronic diary monitoring. Although acceptability and efficacy testing criterion were not fully met, the specific items not met were related to task enjoyment and concentration levels, and to the methodological considerations that would be congruent with a study focused on inferential statistical investigation rather than feasibility assessment. Using the criteria outline by [33], our study demonstrated protocol viability for use of the Tetris intervention in a clinical PTSD sample.

\subsection{Limitations}

Limitations should be considered in interpreting the outcomes of this study. Efficacy-testing outcomes should be interpreted with caution given the lack of a control group, which did not allow for comparison of intrusive memory frequency in the absence of Tetris. Although it is unclear whether changes to intrusive memory frequency were due to imaginal exposure, Tetris, or a combination of both, it does appear that the combination resulted in a small magnitude of reduction in intrusive memory frequency for some people. This is noteworthy if one considers that the initial sessions of exposure therapy could have precipitated a transient increase in intrusive memories such that the Tetris task may have attenuated what may have otherwise been an increase in intrusive memories.

We used a single-case "A-B" experimental design for our efficacy evaluation, such that the within-subjects design did not demand a large sample given the multiple baseline and intervention phase assessments. Nonetheless, we acknowledge that our small sample limits generalizability to other patient groups. We also note that our within-subjects design 
did not include many assessment points before and after the intervention which limited the statistical power of our efficacy-related inferences.

Given our focus on feasibility in this study, we sought to utilise imaginal exposure, a standard component of traumafocused therapy for PTSD [11, 12], as the reactivation cue in order to minimise clinician and patient burden. In non-clinical studies where Tetris gameplay occurred in the memory reconsolidation period, still images were shown for a few seconds $[3,6]$. In Kessler et al.'s [10] clinical study, participants were asked to write the content of their memory on a sheet of paper, whereas Kanstrup et al. [9] used a 'hotspot' form that only included a brief description of the memories. In the current study, imaginal exposure needed to have taken place for at least 15-min prior to Tetris gameplay, however the amount of time needed for reactivation remains unclear and requires further investigation. Identification of an appropriate imaginal exposure period that serves as the reactivation task prior to Tetris gameplay would likely be of benefit in supporting the seamless incorporation of this mechanistic task into psychotherapeutic practice.

Multiple symptom clusters comprise a PTSD diagnosis [40]. It is therefore important that the Tetris intervention not be considered a replacement for psychotherapeutic work that addresses the varying facets of a PTSD diagnosis, but rather as a possible adjunct to existing treatment. However, the use of imaginal exposure as the reactivation cue prior to Tetris gameplay, rather than an additional reactivation task, may make the approach used in the present study a viable option in clinical settings wherein imaginal exposure allows for several of the PTSD symptoms clusters to be addressed. A further limitation of the current study was the absence of registration prior to implementation.

\subsection{Conclusion}

As PTSD has come to be regarded as a disorder of memory [41], identification of approaches that effectively target memory reconsolidation for recovery from PTSD is necessary [42]. Our study aimed to assess the feasibility of using a visuospatial cognitive intervention involving Tetris gameplay as an adjunct intervention to routine treatment for people presenting with PTSD in a hospital inpatient setting. Criteria for feasibility were based on those established by Bowen et al. [33] and were, for the most part, met. However, further investigations are necessary to establish mechanisms of change, the necessary imaginal exposure length, and Tetris gameplay dose (length and frequency). Targeting intrusive memories as a clinical feature appears to be warranted given their role in subsequent PTSD development following trauma exposure $[14,15]$. Our feasibility study contributes to the burgeoning literature investigating the use of cognitive interventions including visuospatial gameplay for trauma-exposed people and is the first to use imaginal exposure as the reactivation task prior to Tetris gameplay, thereby demonstrating support for the use of the Tetris intervention in routine clinical settings.

Acknowledgements This research was supported by an Australian Government Research Training Program Scholarship awarded to Amalia Badawi and by a National Health and Medical Research Council (NHMRC) Early Career Fellowship (GNT1122203) awarded to David Berle. The NHMRC had no role in the design of the study, data collection, data analysis, interpretation of data, or in the writing or revision of the manuscript.

Authors' contributions $A B$ developed the study design and concept under the supervision of, and in consultation with, DB. Testing and data collection were performed by $A B$. AB and DB performed data analysis and interpretation, and drafted the paper. CM assisted with study implementation. CM and ZS provided critical revisions. All authors read and approved the final manuscript.

Data availability The data that support the findings of this study are available on request from the corresponding author. The data are not publicly available due to privacy or ethical restrictions.

\section{Declarations}

Competing interests The authors declared no competing interests with respect to the authorship or the publication of this article.

Open Access This article is licensed under a Creative Commons Attribution 4.0 International License, which permits use, sharing, adaptation, distribution and reproduction in any medium or format, as long as you give appropriate credit to the original author(s) and the source, provide a link to the Creative Commons licence, and indicate if changes were made. The images or other third party material in this article are included in the article's Creative Commons licence, unless indicated otherwise in a credit line to the material. If material is not included in the article's Creative Commons licence and your intended use is not permitted by statutory regulation or exceeds the permitted use, you will need to obtain permission directly from the copyright holder. To view a copy of this licence, visit http://creativecommons.org/licenses/by/4.0/. 


\section{References}

1. Bardo MT, Pentz MA. Translational research. In: APA handbook of research methods in psychology, Vol 2: research designs: quantitative, qualitative, neuropsychological, and biological. Washington: American Psychological Association; 2012. https://doi.org/10.1037/ 13620-029.

2. Badawi A, Berle D, Rogers K, Steel Z. Do cognitive tasks reduce intrusive-memory frequency after exposure to analogue trauma? An experimental replication. Clin Psychol Sci. 2020. https://doi.org/10.1177/2167702620906148.

3. Hagenaars MA, Holmes EA, Klaassen F, Elzinga B. Tetris and Word games lead to fewer intrusive memories when applied several days after analogue trauma. Eur J Psychotraumatol. 2017;8(sup1):1386959. https://doi.org/10.1080/20008198.2017.1386959.

4. Holmes EA, James EL, Coode-Bate T, Deeprose C. Can playing the computer game "Tetris" reduce the build-up of flashbacks for trauma? A proposal from cognitive science. PLoS ONE. 2009;4(1): e4153. https://doi.org/10.1371/journal.pone.0004153.

5. Holmes EA, James EL, Kilford EJ, Deeprose C. Key steps in developing a cognitive vaccine against traumatic flashbacks: visuospatial Tetris versus verbal Pub Quiz. PLoS ONE. 2010;5(11): e13706. https://doi.org/10.1371/journal.pone.0013706.

6. James EL, Bonsall MB, Hoppitt L, Tunbridge EM, Geddes JR, Milton AL, et al. Computer game play reduces intrusive memories of experimental trauma via reconsolidation-update mechanisms. Psychol Sci. 2015;26(8):1201-15. https://doi.org/10.1177/0956797615 583071.

7. Horsch A, Vial Y, Favrod C, Harari MM, Blackwell SE, Watson P, et al. Reducing intrusive traumatic memories after emergency caesarean section: a proof-of-principle randomized controlled study. Behav Res Ther. 2017;94:36-47. https://doi.org/10.1016/j.brat.2017.03.018.

8. Iyadurai L, Blackwell SE, Meiser-Stedman R, Watson PC, Bonsall MB, Geddes JR, et al. Preventing intrusive memories after trauma via a brief intervention involving Tetris computer game play in the emergency department: a proof-of-concept randomized controlled trial. Mol Psychiatry. 2018;23(3):674-82. https://doi.org/10.1038/mp.2017.23.

9. Kanstrup M, Singh L, Göransson KE, Widoff J, Taylor RS, Gamble B, et al. Reducing intrusive memories after trauma via a brief cognitive task intervention in the hospital emergency department: an exploratory pilot randomised controlled trial. Transl Psychiatry. 2021;11(1):30. https://doi.org/10.1038/s41398-020-01124-6.

10. Kessler H, Holmes EA, Blackwell SE, Schmidt A-C, Schweer JM, Bücker A, et al. Reducing intrusive memories of trauma using a visuospatial interference intervention with inpatients with posttraumatic stress disorder (PTSD). J Consult Clin Psychol. 2018;86(12):1076-90. https:// doi.org/10.1037/ccp0000340.

11. American Psychological Association. Clinical practice guideline for the treatment of posttraumatic stress disorder (PTSD) in adults. 2017. https://www.apa.org/ptsd-guideline. Accessed 18 Dec 2021.

12. National Institute for Health and Care Excellence. Post-traumatic stress disorder. 2018. https://www.nice.org.uk/guidance/ng116/chapt er/Recommendations. Accessed 18 Dec 2021.

13. Phoenix Australia-Centre for Posttraumatic Mental Health. Australian guidelines for the treatment of acute stress disorder and posttraumatic stress disorder. 2013. https://www.phoenixaustralia.org/wp-content/uploads/2015/03/Phoenix-ASD-PTSD-Guidelines.pdf. Accessed 18 Dec 2021.

14. Bryant RA, Creamer M, O'Donnell M, Forbes D, McFarlane AC, Silove D, et al. Acute and chronic posttraumatic stress symptoms in the emergence of posttraumatic stress disorder: a network analysis: a network analysis. JAMA Psychiatry. 2017;74(2):135-42. https://doi.org/ 10.1001/jamapsychiatry.2016.3470.

15. Solberg $\varnothing$, Birkeland MS, Blix I, Hansen MB, Heir T. Towards an exposure-dependent model of post-traumatic stress: longitudinal course of post-traumatic stress symptomatology and functional impairment after the 2011 Oslo bombing. Psychol Med. 2016;46(15):3241-54. https://doi.org/10.1017/S0033291716001860.

16. Kandel ER, Dudai Y, Mayford MR. The molecular and systems biology of memory. Cell. 2014;157(1):163-86. https://doi.org/10.1016/j.cell. 2014.03.001.

17. Dudai Y, Morris R. To consolidate or not to consolidate: what are the questions? In: Bolhius JJ, editor. Brain, perception, memory: advances in cognitive neuroscience. Oxford: Oxford University Press; 2000. p. 149-62.

18. McGaugh JL. Memory - a century of consolidation. Science. 2000;287(5451):248-51. https://doi.org/10.1126/science.287.5451.248.

19. Beckers T, Kindt M. Memory reconsolidation interference as an emerging treatment for emotional disorders: strengths, limitations, challenges, and opportunities. Annu Rev Clin Psychol. 2017;13:99-121. https://doi.org/10.1146/annurev-clinpsy-032816-045209.

20. Elsey JWB, Kindt M. Tackling maladaptive memories through reconsolidation: from neural to clinical science. Neurobiol Learn Mem. 2017;142:108-17. https://doi.org/10.1016/j.nlm.2017.03.007.

21. Weathers FW, Blake DD, Schnur PP, Kaloupek DG, Marx BP, Keane TM. Clinician-administered PTSD scale for DSM-5. Boston: National Center for PTSD; 2013.

22. Blue Planet Software. Tetris (UTS Research Version) (Computer game software). Honolulu: Blue Planet Software; 2017.

23. ExpiWell Software. ExpiWell. USA: Purdue University; 2018.

24. Bovin MJ, Marx BP, Weathers FW, Gallagher MW, Rodriguez P, Schnurr PP, et al. Psychometric properties of the PTSD checklist for diagnostic and statistical manual of mental disorders-fifth edition (PCL-5) in veterans. Psychol Assess. 2016;28(11):1379-91. https://doi.org/10. 1037/pas0000254.

25. Weiss DS. The impact of event scale-revised. In: Wilson JP, Keane TM, editors. Assessing psychological trauma and PTSD: a practitioner's handbook. New York: Guilford Press; 2007. p. 168-89.

26. Sundin EC, Horowitz MJ. Impact of event scale: psychometric properties. Br J Psychiatry. 2002;180:205-9. https://doi.org/10.1192/bjp. 180.3.205.

27. Zigmond AS, Snaith RP. The hospital anxiety and depression scale. Acta Psychiatr Scand. 1983;67(6):361-70. https://doi.org/10.1111/j. 1600-0447.1983.tb09716.x.

28. Bjelland I, Dahl AA, Haug TT, Neckelmann D. The validity of the Hospital Anxiety and Depression Scale. An updated literature review. J Psychosom Res. 2002;52(2):69-77. https://doi.org/10.1016/S0022-3999(01)00296-3. 
29. Kessler RC, Andrews G, Colpe LJ, Hiripi E, Mroczek DK, Normand SLT, et al. Short screening scales to monitor population prevalences and trends in non-specific psychological distress. Psychol Med. 2002;32(6):959-76. https://doi.org/10.1017/S0033291702006074.

30. Sampasa-Kanyinga H, Zamorski MA, Colman I. The psychometric properties of the 10-item Kessler Psychological Distress Scale (K10) in Canadian military personnel. PLoS ONE. 2018;13(4): e0196562. https://doi.org/10.1371/journal.pone.0196562.

31. Forbes $D$, Hawthorne $G$, Elliott $P, M c H u g h T$, Biddle $D$, Creamer $M$, et al. A concise measure of anger in combat-related posttraumatic stress disorder. J Trauma Stress. 2004;17(3):249-56. https://doi.org/10.1023/B:JOTS.0000029268.22161.bd.

32. Forbes D, Alkemade N, Mitchell D, Elhai JD, McHugh T, Bates G, et al. Utility of the dimensions of anger reactions-5 (DAR-5) scale as a brief anger measure: research article: utility of DAR-5. Depress Anxiety. 2014;31(2):166-73. https://doi.org/10.1002/da.22148.

33. Bowen DJ, Kreuter M, Spring B, Cofta-Woerpel L, Linnan L, Weiner D, et al. How we design feasibility studies. Am J Prev Med. 2009;36(5):4527. https://doi.org/10.1016/j.amepre.2009.02.002.

34. Lakens D. Calculating and reporting effect sizes to facilitate cumulative science: a practical primer for t-tests and ANOVAs. Front Psychol. 2013;4:863.12. https://doi.org/10.3389/fpsyg.2013.00863.

35. Brossart DF, Laird VC, Armstrong TW. Interpreting Kendall's Tau and Tau-U for single-case experimental designs. Cogent Psychol. 2018;5(1):1518687. https://doi.org/10.1080/23311908.2018.1518687.

36. Baseline corrected tau/Kevin tarlow. Ktarlow.com. http://www.ktarlow.com/stats/tau. Accessed 21 Oct 2021.

37. Tarlow KR. An improved rank correlation effect size statistic for single-case designs: baseline corrected Tau. Behav Modif. 2017;41(4):42767. https://doi.org/10.1177/0145445516676750.

38. Translational Science Spectrum. Nih.gov. 2015. https://ncats.nih.gov/translation/spectrum. Accessed 21 Oct 2021.

39. Eldridge SM, Chan CL, Campbell MJ, Bond CM, Hopewell S, Thabane L, et al. CONSORT 2010 statement: extension to randomised pilot and feasibility trials. BMJ. 2016;355: i5239. https://doi.org/10.1136/bmj.i5239.

40. American Psychiatric Association. Diagnostic and statistical manual of mental disorders [DSM-5 (R)]. 5th ed. Arlington: American Psychiatric Association Publishing; 2013.

41. McNally RJ. Cognitive abnormalities in post-traumatic stress disorder. Trends Cogn Sci. 2006;10(6):271-7. https://doi.org/10.1016/j.tics. 2006.04.007.

42. Hoge CW, Chard KM. A window into the evolution of trauma-focused psychotherapies for posttraumatic stress disorder. JAMA. 2018;319(4):343-5. https://doi.org/10.1001/jama.2017.21880.

Publisher's Note Springer Nature remains neutral with regard to jurisdictional claims in published maps and institutional affiliations. 